This is the final peer-reviewed accepted manuscript of:

Chiara Samorì, Daniele Cespi, Paola Blair, Paola Galletti, Danilo Malferrari, Fabrizio Passarini, Ivano Vassura, and Emilio Tagliavini "Application of switchable hydrophilicity solvents for recycling multilayer packaging materials"; Green Chem., 2017, 19, 1714

The final published version is available online at: http://dx.doi.org/10.1039/C6GC03535C

Rights / License:

The terms and conditions for the reuse of this version of the manuscript are specified in the publishing policy. For all terms of use and more information see the publisher's website.

This item was downloaded from IRIS Università di Bologna (https://cris.unibo.it/)

When citing, please refer to the published version. 


\title{
Application of switchable hydrophilicity solvents for recycling multilayer packaging materials $\uparrow$
}

\author{
Chiara Samori, ${ }^{\star a, b}$ Daniele Cespi, ${ }^{* c, d}$ Paola Blair, ${ }^{b}$ Paola Galletti, ${ }^{a, b}$ Danilo Malferrari, ${ }^{a}$ \\ Fabrizio Passarini, ${ }^{\mathrm{a}, \mathrm{c}}$ Ivano Vassura, ${ }^{\mathrm{a}, \mathrm{c}}$ and Emilio Tagliavini ${ }^{\mathrm{a}, \mathrm{b}}$
}

\section{Introduction}

\begin{abstract}
A new procedure based on switchable hydrophilicity solvents (SHS) was proposed for polyethylene and aluminium recovery from food aseptic packaging. Treatment with $N, N$-dimethylcyclohexylamine (DMCHA) allowed very high material recovery ( $>99 \%$ for aluminium and $>80 \%$ for polyethylene), without compromising the quality in terms of oxidation or polymer degradation. Moreover, the results from a simplified and preliminary life cycle analysis confirm the potential environmental benefits of a SHS approach compared with other treatment and disposal scenarios.
\end{abstract}

Switchable hydrophilicity solvents (SHS) can provide significant improvements related to energy saving and sustainability in the field of extraction processes where high volumes of volatile, flammable, and toxic solvents are usually employed. ${ }^{1}$ SHS, in fact, are peculiar solvents that can reversibly turn their polarity through the addition and removal of $\mathrm{CO}_{2}$, switching from a neutral form (secondary or tertiary amines) into an ionic liquid (ammonium hydrogen carbonate or carbamate). This $\mathrm{CO}_{2}$-promoted switching allows the separation of SHS from the extracted products at the end of the extraction process without consuming energy for evaporation. The application of SHS-based systems has been demonstrated for various separation processes, among which the extraction of lipids or other valuable compounds from vegetable and algal biomasses is very promising. ${ }^{2-5}$ In the field of polymer recovery, Jessop et al. have proposed the use of one of the best performing SHS, $N, N$-dimethylcyclohexylamine (DMCHA), for the recovery of polystyrene foam as a high-density material. ${ }^{6}$

In this paper the application of DMCHA is proposed for the separation and recovery of low density polyethylene (LDPE) and aluminium from multilayer packaging materials. ${ }^{7}$

${ }^{a}$ Centro Interdipartimentale di Ricerca Industriale (CIRI), University of Bologna, via S. Alberto 163, Ravenna, Italy. E-mail: chiara.samori3@unibo.it; Fax: +39-0544-937411; Tel: +39-0544-937353

${ }^{b}$ Department of Chemistry "Ciamician", University of Bologna, via F. Selmi 2, 40126 Bologna, Italy

${ }^{c}$ Department of Industrial Chemistry "Toso Montanari", University of Bologna, viale del Risorgimento 4, 40126 Bologna, Italy. E-mail: daniele.cespi@gmail.com

${ }^{d}$ EMC Innovation Lab S.r.l., Viale Italia 29, 47921 Rimini, Italy

$\dagger$ Electronic supplementary information (ESI) available: Cradle-to-gate inventory for the recovery of 1 ton of composite packaging with different approaches. See DOI: $10.1039 / \mathrm{c} 6 g \mathrm{c} 03535 \mathrm{c}$
Multilayer packaging systems are highly versatile materials largely exploited in the food industry as aseptic packaging for various goods. With the increase of the number of their everyday-life applications, the volume of these laminated products in municipal solid waste has continuously increased and their recyclability has become an environmental and socio-economical issue. These composite materials are made of several laminated layers, including paper $(75 \%$ by weight, composed of long fibres that provide stiffness), aluminium (5\%, which prevents the penetration of air and light, thus assuring the preservation of food contents) and LDPE (20\%, for making the packaging impermeable and for preventing the contact of food with the aluminium layer). The recycling is industrially accomplished by recovering paper fibres by means of a hydra pulping process, whereas the remaining $25 \%$ fraction (LDPE and aluminium) can be treated by agglutination and extrusion to obtain a composite usable in the fabrication of injectionmoulded pellets (polyAl). Recently, several pilot plants or lab techniques have been developed for the separation of LDPE and aluminium; they can be grouped into thermal, mechanical, and chemical processes. The thermal approach (e.g. incineration, plasma $^{8}$ or pyrolysis ${ }^{9,10}$ ) aims at generating energy and at recovering only aluminium, whose quality, however, can be hampered by a high level of oxidation and/or char residue. The mechanical technology is mainly based on exfoliation of the multilayer material with the aid of separating fluids/chemicals such as mixtures of water, surfactants and hydrotropes, ${ }^{11,12}$ organic solvents ${ }^{13}$ or acid solutions. ${ }^{14,15}$ Finally, the chemical strategy foresees the solubilisation of LDPE and the precipitation of aluminium, followed by LDPE recovery by evaporation of the solvent or after the addition of an anti-solvent. The solvents able to effectively dissolve LDPE, however, are few and usually "problematic" (e.g. toluene, xylenes); among them, vegetable oil $^{16}$ represents one of the most environmentally 


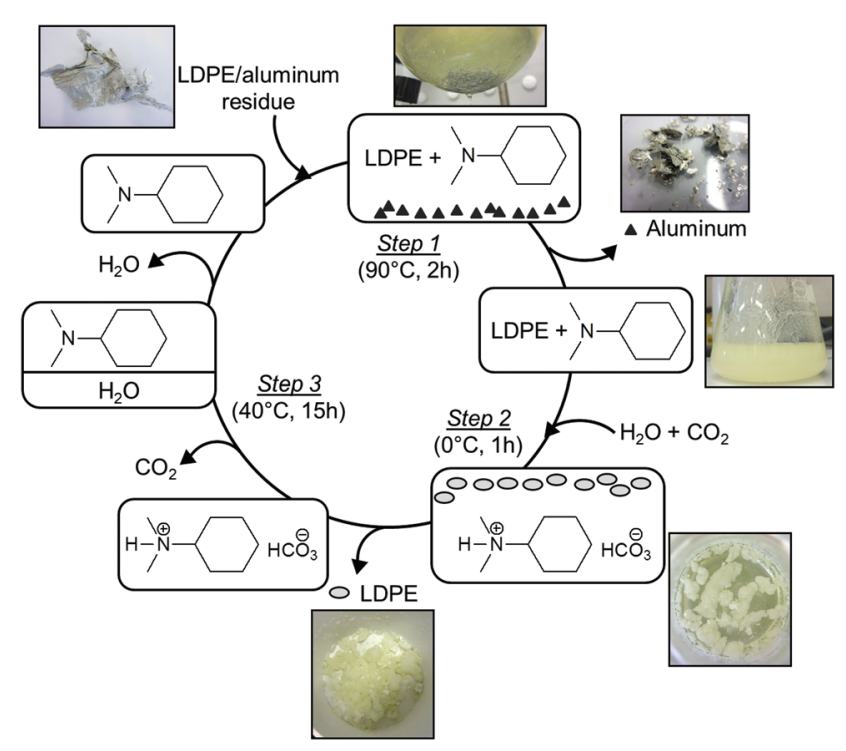

Fig. 1 Process steps and rationale.

friendly options, but chloroform or alcohols are necessary for removing oily contaminations from both LDPE and aluminium. The quality and purity of the outcomes (Al and LDPE), in fact, strongly affect their commercial value, and this holds true especially for the chemical processes for which too harsh conditions can hamper the extrudability of the recovered LDPE or the melting of aluminium flakes.

The protocol presented here (Fig. 1) belongs to the chemical approaches; it aims at treating multilayer packaging waste after the removal of a paper layer (de-papered residues) to separate LDPE and aluminium. Specifically, it exploits DMCHA's peculiarity of tuning its polarity through $\mathrm{CO}_{2}$ addition and removal, switching from a neutral solvent (suitable for LDPE solubilization) to an ionic liquid (in which LDPE is no more soluble and thus can be recovered).

\section{Experimental}

\subsection{Chemicals and materials}

All solvents and chemicals used were obtained from SigmaAldrich (purities $\geq 98 \%$ ) and were used without purification.

\subsection{SHS-process}

Multilayer "de-papered" residue ( $1 \mathrm{~g}$ ) was cut into small pieces (squares of $1 \mathrm{~cm}$ side length) and charged in a round-bottom flask. DMCHA $(80,40,20,10$ or $5 \mathrm{~mL}$, corresponding to a residue/DMCHA ratio of $1.25,2.5,5,10$ and $20 \mathrm{wt} \%$ ) was added and the mixture was heated at $90^{\circ} \mathrm{C}$ (or rt and $50{ }^{\circ} \mathrm{C}$ in the case of $2.5 \mathrm{wt} \%$ ) for $2 \mathrm{~h}$. After that, the "milky" solution of LDPE and DMCHA was separated and residual aluminium was washed with acidic $\mathrm{H}_{2} \mathrm{O}\left(5 \mathrm{~mL}\right.$, achieved by bubbling $\mathrm{CO}_{2}$ in $\mathrm{H}_{2} \mathrm{O}$ until $\mathrm{pH} 5$ ) to remove DMCHA traces. The mixture of LDPE and DMCHA was added to a double volume of $\mathrm{H}_{2} \mathrm{O}$ and cooled in an ice bath; then $\mathrm{CO}_{2}$ was bubbled into the mixture for $1 \mathrm{~h}$ while the flask was vigorously shaken. The floating LDPE was filtered and washed with the same acidic $\mathrm{H}_{2} \mathrm{O}$ used for washing aluminium. Finally, removal of $\mathrm{CO}_{2}$ from the residual water solution was achieved by stirring and heating at $40{ }^{\circ} \mathrm{C}$ overnight. In this way, a complete phase separation was achieved and the organic DMCHA layer was recovered on the top. Each extraction condition was repeated in duplicate.

\subsection{Analysis}

Calcination at $550{ }^{\circ} \mathrm{C}$ for $5 \mathrm{~h}$ was used to determine the amount of aluminium in the de-papered residues; LDPE content was calculated by difference. The residual amount of DMCHA in the recovered aluminium flakes and the elemental composition of recovered LDPE were determined using an elemental analyzer (Thermo Scientific, Flash2000, Organic Elemental Analyzer) by means of the flash combustion technique. FTIR/ATR spectra were recorded on a Nicolet iN10.Mx infrared spectrometer with a germanium crystal. The purity of DMCHA after a switching cycle was detected by ${ }^{1} \mathrm{H}$ NMR spectroscopy by using a $5 \mathrm{~mm}$ probe on a VARIAN Mercury 400 spectrometer in $\mathrm{CDCl}_{3}$. The amount of metal oxides in the recovered aluminium was determined through microwaveassisted acid digestion with hydrochloridric acid (ISO/DIS 14869-3 protocol $)^{17}$ and analyzed by inductively coupled plasma optical emission spectrometry (ICP-OES, ISO 11885:2007 protocol). ${ }^{18}$

\subsection{Life cycle analysis}

Life Cycle Assessment (LCA) is a standardized methodology ${ }^{19,20}$ worldwide recognized as a valuable screening tool to support basic and applied research, in the field of environmental studies and green chemistry. ${ }^{21-24}$ Specifically, its application to the end-of-life management of composite packaging materials represents a consolidated way to evaluate improvements, reached because of a process implementation, or to compare several viable treatment solutions. ${ }^{14,25-27}$ Moreover, companies working in this field well recognized its adoption to implement research choices and disclose the environmental footprints. $^{28-31}$

A simplified life cycle analysis was applied to determine the environmental performances of the SHS-based system described above and make a comparison with three alternative protocols: landfill disposal (a), formic acid treatment (b) and pyrolysis (c). Although disposal procedures are the worst waste treatment solution according to the European Member States (EU waste regulation), ${ }^{32}$ they still are a common procedure in many countries. Therefore, landfill was chosen as a benchmark to simulate the less environmentally-friendly alternative. One ton of multilayer packaging material (paper/PE/Al) sent to treatment was chosen as a functional unit (FU) necessary to refer to the input-output within the system boundaries investigated. ${ }^{19}$ The analysis was limited within the physical boundaries of each treatment plant (Fig. 2), including: (i) all process requests (considering the renewable and fossil resources extraction to satisfy energy and utility needs) and (ii) the main outflows, in terms of emissions and avoided products due to 


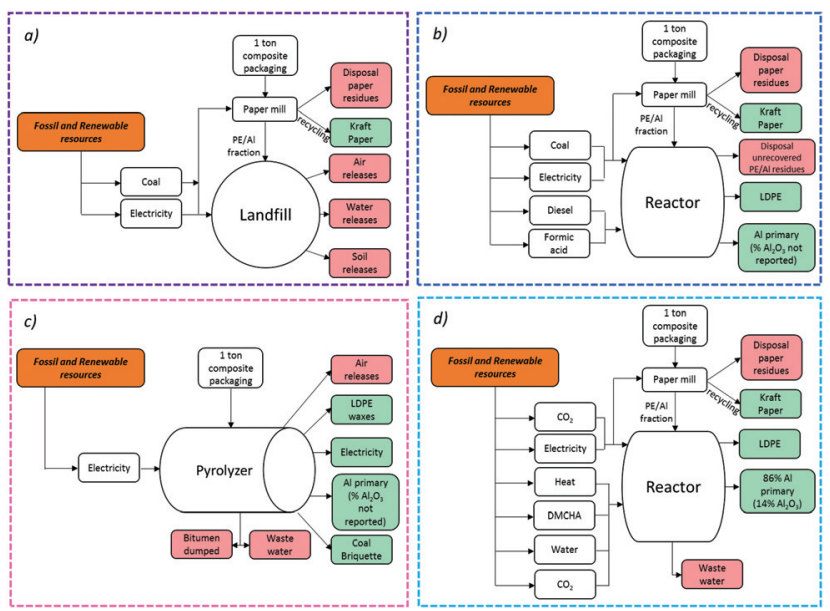

Fig. 2 System boundaries of the four treatment scenarios: (a) landfill, (b) formic acid process, (c) pyrolysis and (d) SHS route.

recoveries. A cradle-to-gate approach was used (the cradle being represented by the set of processes needed to cover plant requirements in terms of auxiliaries and energy, and the gate by all the outputs from the treatment system such as mass/energy recovery and releases into the environment).

The emissions (into air, water, and soil) and production of solid wastes due to each technology have been highlighted with red boxes, whereas the recoveries (avoided processes by LCA) have been highlighted in green. The inventory stage for the SHS-process (main scenario) was completed using primary data collected at the lab scale. In order to make a comparison with other possible scenarios, already published data for a life cycle analysis of the landfill and formic acid processes were adopted. ${ }^{14}$ To the best of our knowledge, no LCA studies on the pyrolysis of the composite packaging have been reported in the literature. Therefore, data from Korkmaz et al. ${ }^{9}$ were modeled to create the input-output model for this scenario, evaluating the energy requirements by enthalpy balance. As depicted by the boundaries, scenarios (a), (b) and (d) assume the disposal or recovery of the LDPE/Al fraction after the treatment of the multilayer packaging material within the paper mill to recover kraft paper. These scenarios include all the inputs/outputs from the paper mill process in terms of mass, energy, emissions and chemicals. On the other hand, scenario (c) was modeled if the entire packaging is used to feed the pyrolyzer, without mill pre-treatment.

The Ecoinvent database ${ }^{33}$ was adopted as a reference library to complete all inventories in terms of energy, auxiliary production, and emissions for all stages involved. Where default processes were not available, dedicated software ${ }^{34,35}$ and literature data ${ }^{36,37}$ were used to fill the gaps (see Tables S1-S4 in the ESI $\dagger$ for the complete inventory of each scenario). Transportation was intentionally omitted due to the lack of reliable data for SHS and pyrolysis processes. In addition, to be more conservative, the same geographical boundaries were assumed, selecting China as a reference country to set up the technologies. The analysis was carried out to address environ- mental concerns at the midpoint level. Six impact categories were chosen among the whole set of eighteen, because of their relevance to the study: climate change (CC), ecotoxicity (ET), fossil fuel depletion (FD), human toxicity (HT), land occupation (LO) and particulate matter formation (PMF). Moreover, the ReCiPe method was selected because of its capability of directly collecting all categories within the same single score indicator (point, Pt) to show the cumulative burden reached by each scenario. SimaPro software (v. 8.0.4.30) ${ }^{38}$ and the ReCiPe method (World H/A, v.1.11) ${ }^{39}$ were used to run the impact assessment stage.

\section{Results and discussion}

\subsection{SHS extraction protocol}

The protocol presented here (Fig. 1) exploits the fact that the neutral form of DMCHA has a low polarity, suitable for solubilizing LDPE at moderate temperature (Step 1), leaving residual aluminium pieces on the bottom of the flask. After the addition of water and $\mathrm{CO}_{2}$ bubbling, the high polarity of the ammonium salt solution causes the separation of apolar LDPE that floats on the surface of the flask (Step 2). Finally, the removal of $\mathrm{CO}_{2}$ regenerates neutral DMCHA as a separate phase from water (Step 3) that can be used for treating another stock of LDPE/aluminium multilayer waste. In the setup of the extraction protocol different temperatures (r.t., $50{ }^{\circ} \mathrm{C}$ and $90{ }^{\circ} \mathrm{C}$ ) and residues/DMCHA ratios $(1.25,2.5,5,10$ and $20 \mathrm{wt} \%$ ) were investigated (Table 1). The solubilization of LDPE did not occur below $90{ }^{\circ} \mathrm{C}$, the temperature at which it was possible to entirely recover the aluminium foil $(>99 \%)$ with all residue/solvent ratios $(1.25,2.5,5$ and $10 \mathrm{wt} \%)$ apart from $20 \mathrm{wt} \%$ : in this case in fact a paste of LDPE/DMCHA formed immediately when the flask was removed from the hot silicon bath, preventing the separation of DMCHA phase from aluminium flakes. After the treatment with DMCHA (Step 1), aluminium was washed with acidic $\mathrm{H}_{2} \mathrm{O}$ (obtained by bubbling $\mathrm{CO}_{2}$ until $\mathrm{pH} 5$ ) to remove residual traces of DMCHA. The DMCHA/LDPE solution was added to $\mathrm{H}_{2} \mathrm{O}$ (volume ratio $1 / 1$ or 1/2) and blown with $\mathrm{CO}_{2}$. After the switching of DMCHA into the corresponding ammonium salt, LDPE separated as floating

Table 1 Tested process parameters, aluminium and LDPE recovery (\%)

\begin{tabular}{lllll}
\hline $\begin{array}{l}\text { Residues/ } \\
\text { DMCHA (wt\%) }\end{array}$ & $\begin{array}{l}T \\
\left({ }^{\circ} \mathrm{C}\right)\end{array}$ & $\begin{array}{l}\text { Al recovery } \\
(\%)\end{array}$ & $\begin{array}{l}\text { LDPE } \\
\text { recovery }(\%)\end{array}$ & $\begin{array}{l}\text { DMCHA/ } \mathrm{H}_{2} \mathrm{O} \\
\text { volume ratio }\end{array}$ \\
\hline 1.25 & 90 & $>99$ & $93 \pm 3$ & $1 / 2$ \\
2.5 & $\mathrm{rt}$ & - & - & - \\
2.5 & 50 & - & - & - \\
2.5 & 90 & $>99$ & $87 \pm 2$ & $1 / 2$ \\
2.5 & 90 & $>99$ & $69 \pm 4$ & $1 / 1$ \\
5 & 90 & $>99$ & $79 \pm 2$ & $1 / 2$ \\
10 & 90 & $>99$ & $72 \pm 16$ & $1 / 2$ \\
20 & 90 & - & - & -
\end{tabular}

${ }^{a}$ The initial amount of aluminium in the treated residues was $25.3 \pm$ $0.3 \mathrm{wt} \%$ (determined by calcination at $550{ }^{\circ} \mathrm{C}$ for $5 \mathrm{~h}$ ); the remaining $75 \mathrm{wt} \%$ was assumed to be LDPE. 
flakes on the surface of the aqueous solution, recovered by filtration (Step 2) and washed with the same acidic $\mathrm{H}_{2} \mathrm{O}$ used for washing aluminium. The recovered LDPE amount ranged from 70 to $90 \%$ (Table 1 ) but it could be increased by reducing mechanical losses due to the small scale of the experiments. The experiment performed with $10 \mathrm{wt} \%$ residue/solvent ratio was scarcely reproducible in terms of LDPE recovery because a viscous solution of DMCHA and LDPE was formed at rt, preventing an efficient recovery. The lowest recovery (69\%) was obtained when the DMCHA $/ \mathrm{H}_{2} \mathrm{O}$ volume ratio was $1 / 1$ instead of $1 / 2$ (in the case of $1 / 1$, LDPE separated in a "paste" mushy form, difficult to filter).

The switching back of the $\mathrm{DMCHAH}^{+} \mathrm{HCO}_{3}{ }^{-}$ammonium salt into DMCHA (Step 3) was achieved by stirring and heating at $40{ }^{\circ} \mathrm{C}$ the aqueous solution. ${ }^{40}$ At the end of this process, the amount of DMCHA recovered as separated, water insoluble phase was $80 \%$; for achieving better amine separation, an increase of $\mathrm{pH}$ by addition of $\mathrm{KOH}$ was necessary. At $\mathrm{pH} 11$, more than $96 \%$ of the initial amine could be recovered.

The recovered DMCHA did not contain detectable impurities (checked by ${ }^{1} \mathrm{H}$ NMR spectroscopy) and thus it could be used without any further purification for another extraction cycle.

\subsection{Aluminium and LDPE characterization}

Fourier transform infrared (FTIR) attenuated total internal reflection (ATR) spectra showed that on both faces of recovered Al pieces, residual LDPE was still present (Fig. 3): the bands related to the symmetric and asymmetric stretching modes of $\mathrm{CH}_{2}$ (2916 and $2847 \mathrm{~cm}^{-1}$ ) were clearly detectable as well as the bending and rocking modes of $\mathrm{CH}_{2}$ at about $1460 \mathrm{~cm}^{-1}$ and $725 \mathrm{~cm}^{-1}$, respectively.

The contamination by LDPE of the recovered aluminium pieces (Table 2) decreased with increasing DMCHA to LDPE-Al ratio; the aluminium obtained from the most diluted extraction system (1.25 wt\%) contained less LDPE (below 2\%), around 4 times lower than what was achieved from the $10 \mathrm{wt} \%$ system.

No traces of nitrogen were detected on the recovered $\mathrm{Al}$, confirming that washing with acidic $\mathrm{H}_{2} \mathrm{O}$ effectively removed DMCHA traces as an ammonium salt. The percentage of metallic aluminium in the recovered material, a crucial parameter

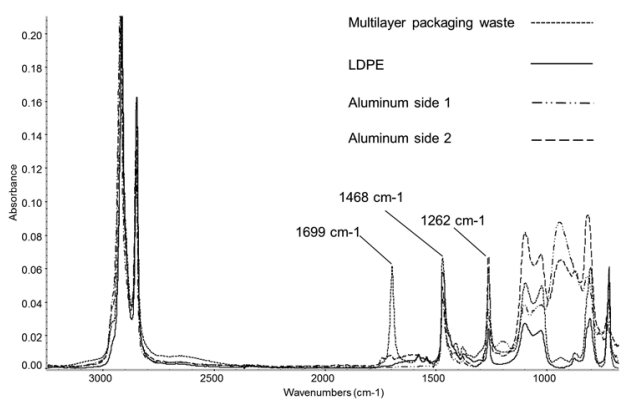

Fig. 3 ATR spectra of multilayer "de-papered" residue, recovered aluminium pieces and recovered LDPE.
Table 2 Contamination detected in the recovered aluminium and LDPE ( $\mathrm{C}$ and $\mathrm{N} \%$ determined by elemental analysis)

\begin{tabular}{lllll}
\hline $\begin{array}{l}\text { Residue/ } \\
\text { DMCHA (wt\%) }\end{array}$ & $\begin{array}{l}\text { N in } \\
\text { LDPE (\%) }\end{array}$ & $\begin{array}{l}\text { DMCHA cont. } \\
\text { in LDPE (wt\%) }\end{array}$ & $\begin{array}{l}\mathrm{C} \text { in } \\
\mathrm{Al}(\%)\end{array}$ & $\begin{array}{l}\text { LDPE cont. } \\
\text { in Al (wt\%) }\end{array}$ \\
\hline 1.25 & $0.21 \pm 0.02$ & 1.9 & $1.6 \pm 0.1$ & 1.9 \\
2.5 & $0.12 \pm 0.04$ & 1.1 & $2.4 \pm 0.3$ & 2.8 \\
$2.5^{a}$ & $0.14 \pm 0.05$ & 1.3 & $2.4 \pm 0.6$ & 2.8 \\
5 & $0.13 \pm 0.05$ & 1.2 & $4.5 \pm 0.3$ & 5.2 \\
10 & $0.40 \pm 0.01$ & 3.9 & $7.0 \pm 0.2$ & 8.1
\end{tabular}

${ }^{a} \mathrm{DMCHA} / \mathrm{H}_{2} \mathrm{O}$ volume ratio of $1 / 1$.

for determining its commercial value after an industrial remelting process, was $86 \%$. This high value confirmed the nonoxidative behavior of DMCHA in the developed process.

The contamination by DMCHA in LDPE, calculated from the amount of $\mathrm{N}$ detected in the recovered LDPE, was low (around 1-2 wt\%). The highest value of DMCHA (4 wt\%) was obtained in the case of $10 \mathrm{wt} \%$ residue/DMCHA ratio, indicating that the viscous DMCHA/LDPE solution formed at $\mathrm{rt}$ at the end of the experiment affected both the recovery and the separation of DMCHA from LDPE itself. The recovered LDPE had similar thermostability properties to standard LDPE polymers when subjected to a thermal degradation in air (Fig. 4): its weight was stable until $250{ }^{\circ} \mathrm{C}$ (weight losses $<0.1 \%$ ), with a strong weight loss between 250 and $410^{\circ} \mathrm{C}$; the main difference with standard LDPE polymers was a solid residue ( $4.5 \mathrm{wt} \%$ ) obtained at the end of the thermal treatment $\left(600^{\circ} \mathrm{C}\right)$. This finding confirms that the treatment with DMCHA did not alter significantly the quality of both aluminium and LDPE.

\subsection{Life cycle assessment}

Table 3 and Fig. 5 depict the main results from the impact assessment stage. The overall single scores indicated that when technologies are based on mass and/or energy recovery (formic acid, pyrolysis, and SHS-route), the avoided impacts largely exceed the potential burdens on the environment (most LCA studies refer to the avoided impacts using negative scores: the higher the absolute values, the greater the benefits). On the contrary, in the case of landfill the potential benefits are

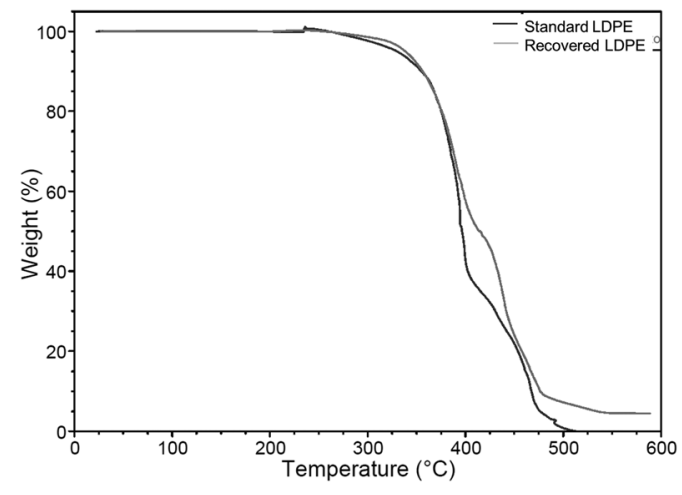

Fig. 4 Comparison of TGA profiles of recovered and standard LDPE. 
Table 3 Results of the cradle-to-gate analysis per impact category: climate change (CC); human toxicity (HT); particulate matter formation (PMF); environmental toxicity (ET); land occupation (LO); fossil fuel depletion (FD)

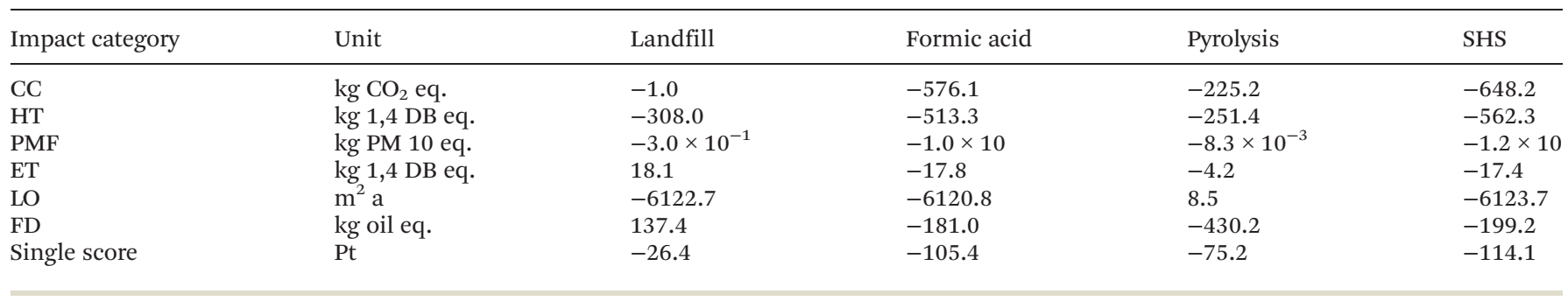

\begin{tabular}{|ll}
\hline$\square$ Climate change & $\square$ Human toxicity \\
$\square$ Particulate matter formation & $\square$ Ecotoxicity \\
$\square$ Land occupation & $\square$ Fossil fuels depletion \\
\hline
\end{tabular}

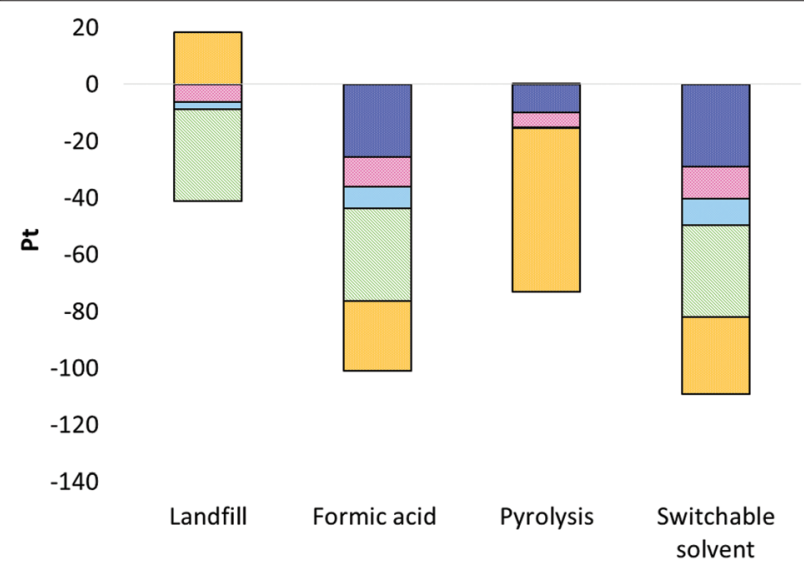

Fig. 5 Cradle-to-gate analysis in terms of ReCiPe single score.

associated with the paper recycling at the mill only, since the dumping procedure does not lead to environmental impacts mitigation (no mass and energy recovery). However, even if the recycled paper produces benefits in terms of HT, PMF and LO, it leads to negative effects in terms of FD due to the mill requirements: around $301 \mathrm{~kg}$ of coal and $379 \mathrm{kWh}$ per ton of composite packaging. ${ }^{14}$ As further confirmation of the benefits due to paper recycling, impacts on LO have quite similar trends for each scenario in which a $\mathrm{PE} / \mathrm{Al}$ fraction treatment follows the mill procedures. Pyrolysis requires only an energetic input and it can recover both electricity and coal briquettes.

These energetic recoveries lead to a higher reduction in terms of FD, estimated at around $-313 \mathrm{~kg}$ of oil eq. of the total -430 ( $64 \%$ from electricity and the rest from briquettes), and for the PMF category (around $-3.0 \mathrm{~kg}$ of PM10 eq.). Reduction of PMF is mainly driven by the lower amount of substances emitted during fuel combustion to produce process steam at thermoelectric plants. The energetic recoveries lead to relevant benefits also in terms of $\mathrm{CC}\left(-1077 \mathrm{~kg}\right.$ of $\mathrm{CO}_{2}$ eq. $)$ and $\mathrm{HT}$ (-266 kg 1,4 DB eq.). Additionally, since metallic aluminium and good- $M_{\mathrm{w}}$ (molecular weight) LDPE waxes are obtained from pyrolysis, ${ }^{9}$ a replacement of virgin materials can also be assumed, providing additional benefits for the categories of $\mathrm{CC}\left(-832\right.$ and $-392 \mathrm{~kg}$ of $\mathrm{CO}_{2}$ eq.), $\mathrm{FD}(-195$ and $-293 \mathrm{~kg}$ oil eq.) and $\mathrm{HT}$ ( -341 and $-5 \mathrm{~kg} \mathrm{1,4} \mathrm{DB}$ eq.) (scores for $\mathrm{Al}$ and LDPE, respectively). Data about the percentage of oxidized aluminium $\left(\mathrm{Al}_{2} \mathrm{O}_{3}\right)$ have not been reported in the literature.

Cumulative impact achieved by pyrolysis was estimated at around $-75 \mathrm{Pt}$, lower than the environmental benefits achieved by the formic acid process $(-105 \mathrm{Pt})$.

On the other hand, although formic acid treatment is more resource-consuming (coal, diesel, electricity, and formic acid) and leads to a lower amount of recovered aluminium $(36 \mathrm{~kg} v s$. $49 \mathrm{~kg}$ ), it guarantees greater LDPE recycling (196 kg vs. $125 \mathrm{~kg}$ ), influencing the $\mathrm{CC}$ and $\mathrm{FD}$ for $-411 \mathrm{~kg}$ of $\mathrm{CO}_{2}$ eq. and $-307 \mathrm{~kg}$ oil eq. Also in this case, data about the percentage of oxidized aluminium $\left(\mathrm{Al}_{2} \mathrm{O}_{3}\right)$ have not been reported in the literature. Differently from the thermal valorization of the entire packaging waste, the combined cycle (also including paper recovery) saves $660 \mathrm{~kg}$ of kraft paper per ton treated. This last saving avoids the paper production from virgin cellulose, with a great effect on LO $\left(-6121 \mathrm{~m}^{2}\right.$ subtracted), CC $(-558 \mathrm{~kg}$ of $\mathrm{CO}_{2}$ eq.) and $\mathrm{FD}(-164 \mathrm{~kg}$ oil eq.). This combination leads to greater cumulative benefits of formic acid-based treatment, when compared with the pyrolysis.

Although the amount of kraft paper recovered is the same, overall scores reached by the SHS-route are quite higher in terms of avoided impacts $(-114 \mathrm{Pt})$. A further analysis (Fig. S1 in the ESI†) run for the recovery treatments alone (landfill is not included) reveals that the main contributions to the impacts mitigation are due to the LO (28\%), CC (25\%) and the avoided usage of fossil fuels (24\%). In fact, recovering LDPE and $\mathrm{Al}$ prevents their production from virgin raw materials and guarantees a saving of $-389 \mathrm{~kg}$ of oil eq. per ton treated. A reduction greater than $-267 \mathrm{~kg}$ of oil eq. (around $70 \%$ contribution) is achieved thanks to the avoided manufacture of $187 \mathrm{~kg}$ of virgin LDPE, in which fossil-based resources are used both in the production of energy and feedstocks. Fig. S2a and $\mathrm{b}$ in the ESI† shows the network of the results for FD and CC categories. The avoided impacts are depicted using green arrows: the thicker the flows, the higher the avoided burden to the global scores. On the contrary, red arrows reflect the opposite situation of increasing the burdens. Since the Al recovery achieved by using a SHS-approach was close to $99 \%$, and $86 \%$ is metallic $\mathrm{Al}$, around $43 \mathrm{~kg}$ of primary $\mathrm{Al}$ are recovered with a contribution of $-122 \mathrm{~kg}$ oil eq. (Fig. S2a $\dagger$ ) (14\% of aluminium 
is obtained in an oxidized form with no sensible environmental and economic contribution). These results highlight the importance of recovering aluminium in the primary form, because of the intense energy requirements during its manufacturing chain, evaluated at around $190-230 \mathrm{MJ} \mathrm{kg}^{-1} \cdot{ }^{41}$ The energy saving could have relevant benefits also in terms of environmental issues. Looking at the contribution to the CC, $-521 \mathrm{~kg}$ of $\mathrm{CO}_{2}$ eq. are due to the recovery of virgin Al. This trend is well depicted by Fig. S2b in the ESI, $\dagger$ which confirms a contribution of $80 \%$ of the overall value for the greenhouse gas (GHG) emissions ( $-648 \mathrm{~kg}$ of $\mathrm{CO}_{2}$ eq.). In both cases (FD and $\mathrm{CC}$ ), the networks depict that the main incidence is related to the electricity consumptions involved in the electrolysis, a process necessary to obtain the virgin metal. The energy saving is also the main driver for the avoided impacts on the PMF and HT categories ( $8 \%$ and $10 \%$ contribution on the overall single score, see Fig. S1 in the ESI $\dagger$ ). In fact, the prevented release of gases and particulates, on the one hand, and of heavy metals on the other (mainly due to the disposal procedures within the entire chain) leads to sensible reductions in both categories. Inventories of the avoided substances, together with the percentage contributions, are reported in Table S5 in the ESI. $\dagger$

Even if the results from the simplified LCA show a positive trend for the SHS process, some considerations are mandatory. First, landfill represents a common option and the recovery with formic acid is already developed in China. This means that the Technology Readiness Levels (TRLs) for these two scenarios are higher than the invention presented in this paper, only developed on the laboratory scale. Then, the LCA should be considered as a good approximation of a simplified early stage analysis, carried out using preliminary data provided by experiments (automatically affected by uncertainties). However, different from the formic acid technology, the SHSroute was proposed in Italy. Therefore, assuming the same energy requirements, but substituting the China electricity mix with the Italian one and assuming the replacement of coal (used in the paper mill) with natural gas, a greater reduction up to $-142 \mathrm{Pt}$ could be achieved. This is a further confirmation of the potentiality of the SHS-route proposed here, which represents an interesting alternative to recover value from composite packaging.

\section{Conclusion}

The SHS system based on a lipophilic tertiary amine, such as DMCHA, can be successfully applied for processing multilayer packaging materials with the aim of recovering both LDPE and aluminium. The use of such systems was highly effective, without compromising the quality of the recovered materials; this holds true particularly for $\mathrm{Al}$, whose recovery rate was $>99 \%$, with a great efficiency for the metallic form $(86 \%)$. The mild process conditions (room pressure and temperature below $100{ }^{\circ} \mathrm{C}$ ) and the possibility of recycling the solvent just by adding and removing $\mathrm{CO}_{2}$ underline the greenness of the entire process. The results achieved by the simplified life cycle analysis in comparison with other waste treatment technologies confirmed this finding: the overall reduction of the global environmental load could vary from $-8 \%$ up to $-35 \%$ if a different energy mix was adopted. Several interesting aspects came out from a preliminary environmental assessment; a further analysis on the pilot scale is mandatory to verify in detail all the economic and environmental advantages and drawbacks associated with the process. However, the results presented in this paper represent further confirmation of the importance of the Green Chemistry principles at the early design stage to minimize the production of waste (the use of a recyclable solvent) and increase the process efficiency.

\section{Acknowledgements}

We thank Emilio Catelli and Francesca Volpi of the University of Bologna, Italy, for FTIR/ATR spectra and Dr Laura Mazzocchetti of the University of Bologna, Italy, for the TGA measurements. We thank Elena Tommasi of the University of Bologna, Italy, for helpful assistance in lab experiments. We thank ERRE QUADRO s.r.l., Italy, for the analysis of metal oxides in the recovered aluminium. We thank the University of Bologna (RFO programme) and Fondo Europeo per lo Sviluppo Regionale (POR-FESR 2007-2013) for funding.

\section{Notes and references}

1 J. R. Vanderveen, J. Durelle and P. G. Jessop, Green Chem., 2014, 16, 927.

2 L. Phan, H. Brown, J. White, A. Hodgson and P. G. Jessop, Green Chem., 2009, 11, 53.

3 D. Fu, S. Farag, J. Chaouki and P. G. Jessop, Bioresour. Technol., 2014, 154, 101.

4 C. Samorì, D. López Barreiro, R. Vet, L. Pezzolesi, D. W. F. Brilman, P. Galletti and E. Tagliavini, Green Chem., 2013, 15, 353.

5 C. Samorì, L. Pezzolesi, D. López Barreiro, P. Galletti, A. Pasteris and E. Tagliavini, RSC Adv., 2014, 4, 5999.

6 P. G. Jessop, L. Kozycz, Z. G. Rahami, D. Schoenmakers, A. R. Boyd, D. Wechsler and A. M. Holland, Green Chem., 2011, 13, 619.

7 E. Tagliavini, P. Galletti, C. Samorì and D. Malferrari, Metodo per separare e recuperare polietilene e alluminio da un materiale poliaccoppiato, Pat. Pend, 102016000114545, 2016, University of Bologna (IT).

8 F. L. Neves, O Papel, 1999, 61, 38.

9 A. Korkmaz, J. Yanik, M. Brebu and C. Vasile, Waste Manage., 2009, 29, 2836.

10 J. Haydary, D. Susa and J. Dudáš, Waste Manage., 2013, 33, 1136.

11 S. Kernbaum and H. Seibt, US Patent, 0319618A1, 2013.

12 F. Lovis, H. Seibt and S. Kernbaum, WO Patent, 169801A1, 2015. 
13 S. Zhang, K. Luo, L. Zhang, X. Mei, S. Cao and B. Wang, J. Chem. Technol. Biotechnol., 2015, 90, 1152.

14 M. Xie, Q. Qiao, Q. Sun and L. Zhang, Int. J. Life Cycle Assess., 2013, 18, 626.

15 D. Yan, Z. Peng, Y. Liu, Q. Huang, M. Xie and Q. Wang, Waste Manage., 2015, 35, 21.

16 J. E. Rodríguez-Gómez, Y. Q. Silva-Reynoso, V. VarelaGuerrero, A. Núñez-Pineda and C. E. Barrera-Díaz, Fuel, 2015, 149, 90.

17 ISO/DIS 14869-3 Dissolution for the determination of the total element content, Part 3: Dissolution with hydrofluoric, hydrochloric and nitric acids using the pressurised microwave technique, International Organization for Standardization, Geneva, Switzerland, 2015.

18 ISO 11885 Determination of selected elements by inductively coupled plasma optical emission spectrometry (ICP-OES), International Organization for Standardization, Geneva, Switzerland, 2007.

19 EN ISO 14040 Environmental Management, Life Cycle Assessment, Principles and Framework, International Organization for Standardization, Geneva, Switzerland, 2006.

20 EN ISO 14044 Environmental Management, Life Cycle Assessment, Requirements and Guidelines, International Organization for Standardization, Geneva, Switzerland, 2006.

21 D. Kralisch, D. Ott and D. Gericke, Green Chem., 2015, 17, 123.

22 D. Cespi, F. Passarini, G. Mastragostino, I. Vassura, S. Larocca, A. Iaconi, A. Chieregato, J.-L. Dubois and F. Cavani, Green Chem., 2015, 17, 343.

23 D. Cespi, E. S. Beach, T. E. Swarr, F. Passarini, I. Vassura, P. J. Dunn and P. T. Anastas, Green Chem., 2015, 17, 3390.

24 D. Cespi, F. Passarini, I. Vassura and F. Cavani, Green Chem., 2016, 18, 1625.

25 A. L. Mourad, E. E. C. Garcia, G. B. Vilela and F. Von Zuben, Resour., Conserv. Recycl., 2008, 52, 678.

26 L. Mourad, E. E. C. Garcia, G. B. Vilela and F. Von Zuben, Int. J. LCA, 2008, 13, 140.

27 M. Xie, W. Bai, L. Bai, X. Sun, Q. Lu, D. Yan and Q. Qiao, J. Cleaner Prod., 2016, 112, 4430.

28 Tetra Pak Inc. and Oestfold Research Foundation, Investigating the life-cycle environmental profile of liquid food packaging systems, 1999, http://www.tetrapak.com/it/ sustainability/managing-our-impact/climate-impact/life-cycleassessments (accessed November 2016).

29 Tetra Pak Inc., Life cycle inventory of container systems for wine, 2006, http://www.tetrapak.com/it/sustainability/managing-our-impact/climate-impact/life-cycle-assessments (accessed November 2016).

30 Fachverband Kartonverpackungen für flüssige Nahrungsmittel e.V. (FKN), Life cycle assessment: beverage cartons under test, 2007, http://www.tetrapak.com/it/sustainability/managing-our-impact/climate-impact/life-cycleassessments (accessed November 2016).

31 Bio Intelligence Service S.A.S., Nordic Life Cycle Assessment Wine Packaging Study, 2010, http://www. tetrapak.com/it/sustainability/managing-our-impact/climateimpact/life-cycle-assessments (accessed November 2016).

32 Directive 2008/98/EC of the European Parliament and of the Council of 19 November 2008 on waste and repealing certain directives.

33 Ecoinvent Centre (formerly Swiss Centre for Life Cycle Inventories) (2016) Ecoinvent 3.1 Database.

34 G. Wernet, S. Hellweg, U. Fischer, S. Papadokonstantakis and K. Hungerbühler, Environ. Sci. Technol., 2008, 42, 6717.

35 G. Wernet, S. Papadokonstadakis, S. Hellweg and K. Hungerbühler, Green Chem., 2009, 11, 1826.

36 N. von der Assen, J. Jung and A. Bardow, Energy Environ. Sci., 2013, 6, 2721.

37 N. von der Assen, P. Voll, M. Peters and A. Bardow, Chem. Soc. Rev., 2014, 43, 7982.

38 PRé Consultants, SimaPro, PhD version 8.0.4.30, Amersfoort, The Netherlands, 2016.

39 M. Goedkoop, R. Heijungs, M. Huijbregts, A. De Schryver, J. Struijs and R. van Zelm, ReCiPe 2008 - A life cycle impact assessment method which comprises harmonised category indicators at the midpoint and the endpoint level, First edition (version 1.08), Ministry of Housing, Spatial Planning and the Environment (VROM), Netherlands, 2013.

40 P. G. Jessop, L. Kozycz, Z. Ghoshouni Rahami, D. Schoenmakers, A. R. Boyd, D. Wechsler and A. M. Holland, Green Chem., 2011, 13, 619.

41 M. J. Eckelman, G. M. Mudd and T. E. Norgate, Metals and energy, in Environmental Impacts of Metals, ed. E. Van der Voet, UNEP Resource Panel, 2013. 